\title{
OPTIMUM ENERGY MANAGEMENT OF PEM FUEL CELL SYSTEMS BASED ON MODEL PREDICTIVE CONTROL
}

\author{
Chrysovalantou Ziogou $^{(1)}$, Michael C. Georgiadis ${ }^{(2)}$, Spyros Voutetakis $^{(1)}$, Simira Papadopoulou $^{(3)}$ \\ (1) Chemical Process and Energy Resources Institute (CPERI), Centre for Research and Technology Hellas (CERTH), \\ Thessaloniki, Greece,Email: cziogou@cperi.certh.gr,paris@cperi.certh.gr \\ ${ }^{(2)}$ Department of Chemical Engineering, Aristotle University of Thessaloniki, Thessaloniki, Greece, \\ Email:mgeorg@auth.gr \\ (3) Department of Automation Engineering, Alexander Technological Educational Institute of Thessaloniki, \\ Thessaloniki, Greece, Email: shmira@autom.teithe.gr
}

\begin{abstract}
This work presents an optimum energy management framework, which is developed for integrated Polymer Electrolyte Membrane (PEM) fuel cell systems. The objective is to address in a centralized manner the control issues that arise during the operation of the fuel cell (FC) system and to monitor and evaluate the system's performance at real time. More specifically the operation objectives are to deliver the demanded power while operating at a safe region, avoiding starvation, and concurrently minimize the fuel consumption at stable temperature conditions. To achieve these objectives a novel Model Predictive Control (MPC) strategy is developed and demonstrated. A semiempirical experimentally validated model is used which is able to capture the dynamic behaviour of the PEMFC. Furthermore, the MPC strategy was integrated in an industrial-grade automation system to demonstrate its applicability in realistic environment. The proposed framework relies on a novel nonlinear MPC (NMPC) formulation that uses a dynamic optimization method that recasts the multivariable control problem into a nonlinear programming problem using a warm-start initialization method and a search space reduction technique which is based on a piecewise affine approximation of the variable's feasible space.

The behaviour of the MPC framework is experimentally verified through the online deployment to a small-scale fully automated PEMFC unit. During the experimental scenarios the PEMFC system demonstrated excellent response in terms of computational effort and accuracy with respect to the control objectives.
\end{abstract}

\section{INTRODUCTION AND MOTIVATION}

The shift towards a low carbon, efficient and secure economy requires targeted deployment of innovative technologies and increased exploitation of renewable energy sources. Furthermore, it is recognized that a technological shift and the development of new clean technologies are vital for a successful transition to a decarbonized and sustainable future economy. Although a number of diverse technologies exist that aim at the same target, the synergy between the increased use of renewable energy sources, renewable hydrogen and electricity from fuel cells represent one of the promising ways to realize sustainable energy. Fuel cell and hydrogen technologies have the potential to contribute to the ambitious energy and climate objectives of the European Union for 2020 which are the reduction of the greenhouse gas emissions by $20 \%$, the increase of the share of renewable energy to $20 \%$ and the improvement of the energy efficiency by $20 \%$ [1].

\subsection{Importance of Control Strategies to FC systems}

In order to take advantage of FC's great potential a number of technological challenges must be addressed that require significant efforts of research and development. Continuous research efforts are imperative for the realization of FC system-level goals for performance, predictability, stability, and other properties through appropriate analysis, design and implementation. In this context, the optimum energy management through appropriate control methods can function as a catalyst that transforms technological innovation to systems engineering novelties.

The impact of control technology is evident in a wide range of application areas, including fuel cells, as it is the necessary facilitator for achieving desired objectives and fulfilling application-specific goals [2]. Fuel cell systems exhibit fast dynamics, nonlinearities and uncertainties that constitute challenges requiring appropriate control in order to be confronted effectively. The use of efficient control strategies would not only increase the performance of these systems, but would increase the number of operational hours as their lifetime is preserved by operating at optimal levels and also reduce the cost per produced kilowatt-hour. Overall control can be considered as an enabling technology for the optimum energy management of fuel cell systems. Based on this motivation initially the experimental system is described along with the basic modelling approach (Section 2). Subsequently the advanced control (Section 3) and structure of the online energy management framework is discussed (Section 4) which is deployed to the PEMFC into consideration. 


\section{PEM FUEL CELL EXPERIMENTAL SETUP AND MODELING}

In this work a small-scale fully automated fuel cell unit was used which was designed and constructed at CPERI/CERTH. Fig. 1 presents the simplified process and instrumentation (P\&ID) diagram of the experimental PEMFC along with the measured variables and the control actions that will be determined by the the advanced control scheme. The variables that constitute the Input Output (I/O) field of the unit are acquired by a Supervisory control and Data Acquisition (SCADA) system. The PEM fuel cell is by Electrochem ${ }^{\circledR}$, has a Nafion 1135 ( $89 \mu \mathrm{m}$ thickness) membrane electrode assembly (MEA) with an active area of $25 \mathrm{~cm}^{2}$, while the power is drawn from the fuel cell through a DC load (KIKUSUI $\left.{ }^{\circledR}\right)$. The air and the hydrogen are supplied from pressurized cylinders by two mass flow controllers (MFCs). Hydrators and heated lines are used to provide the proper humidity of the inlet gases. The temperature is maintained by an air cooling subsystem and an electrical resistance.

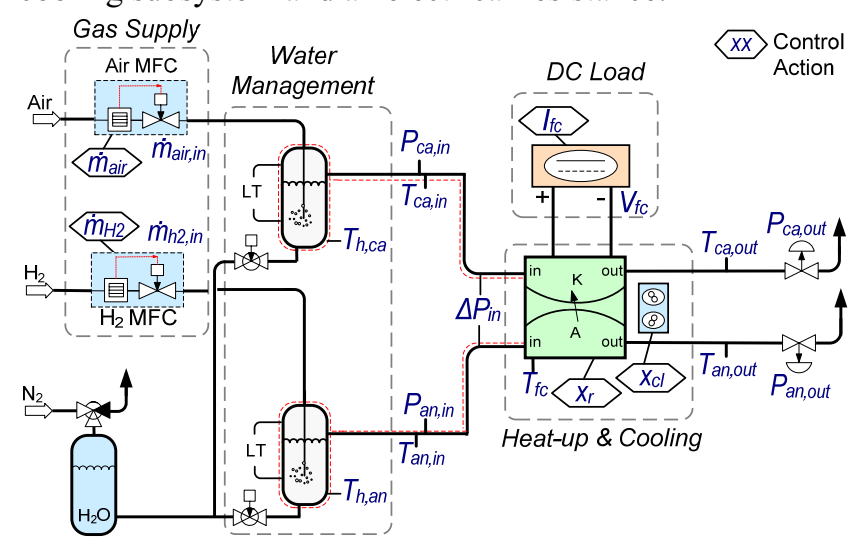

Figure 1. Simplified P\&ID of CERTH/CPERI's PEMFC unit

The objective is to manage the produced energy by proper manipulation of the four distinct, yet interacting, subsystems which are related to the power production, the gas supply, the temperature and the water management. Each subsystem has its own control objective and a number of sensors and actuators are used to monitor the system's behavior.

The inlet gasses, the FC temperature and the power are controlled by the proposed model-based framework while the hydrator's temperature and the heated lines are controlled by conventional PIs due to their slow dynamic behaviour. All other system components (pumps, heaters, valves, etc) are controlled by the SCADA system.

\subsection{Nonlinear Dynamic Model}

The fuel cell unit (Fig. 1) was modelled by an experimentally validated semi-empirical nonlinear dynamic model [3] which considers the mass dynamics in the gas flow channels, the gas diffusion layers (GDL) and the membrane. Also the respective electrochemical equations are used for the calculation of the voltage and an energy balance differential equation is integrated to capture the temperature evolution. An illustrative representation of the involved variables and main components of the PEMFC model is show at Fig. 2.

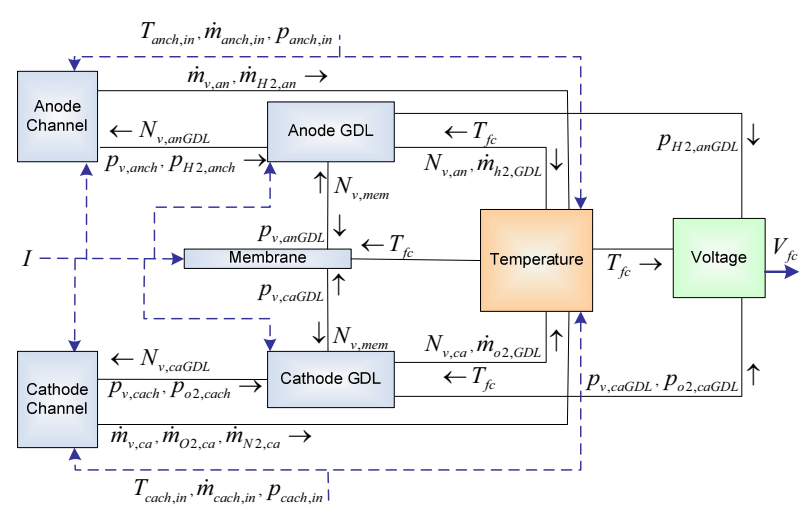

Figure 2. PEMFC mathematical model structure

The developed model couples both the theoretical validity (mechanistic equations) and the inherent simplicity of the empirical application (semi-empirical equations). The described dynamic model is not system dependent as it can be adjusted to describe any other PEM fuel cell system by performing a number of sequentially executed actions. These actions are determination of

- the physical characteristics of the PEM fuel cell,

- the unit specific parameters

- the empirical parameters utilized by the electrical subsystem of the model.

The value of the empirical parameters are determined by a systematic parameter estimation procedure as long as experimental data or data from the manufacturer are provided.

\section{ADVANCED MODEL-BASED PREDICTIVE CONTROL STRATEGIES}

The objective of advanced control strategies is to achieve a set of predefined conditions for the process and maintain the operation at the desired or optimal values [4]. Model predictive control (MPC) also known as receding horizon control (RHC), is becoming a preferred control strategy for a large number of industrial processes [5]. The main reasons for the increased popularity include the ability to handle state and input constraints, dynamic nonlinearities of the process into consideration and simultaneous satisfaction of economical and operating constraints under an integrated optimization methodology. Moreover recent advances in optimization enable the move towards direct online optimizing control. These characteristics in combination with the demanding operation of a PEMFC, signify that the application of NMPC to such 
systems is a suitable approach. In the current work an MPC formulation is selected to effectively handle the interrelated control objectives of a PEMFC.

In general MPC refers to a methodology which makes explicit use of a process model to optimize the future predicted behavior of a process. The main objective is to obtain control actions that minimize a cost function related to selected objectives or performance indices of the system. At each sampling time an optimal control problem is solved using measurements acquired from the system and it yields the appropriate control inputs for the system.

MPC computes online a finite-time constrained optimization problem over a prediction horizon $\left(T_{p}\right)$, using the current state of the process as the initial state. The optimization yields an optimal control sequence $\left(u_{k} . . u_{k+N c}\right)$ over a control horizon $\left(T_{c}\right)$, which is partitioned into $N_{c}$ intervals and only the first control action $\left(u_{k}\right)$ for the current time is applied to the system. We consider the following formulation of the MPC problem [6]:

$$
\begin{aligned}
& \min _{u} J=\sum_{j=1}^{N p}\left(\hat{y}_{k+j}-y_{s p, k+j}\right)^{T} Q R\left(\hat{y}_{k+j}-y_{s p, k+j}\right)+\sum_{l=0}^{N c-1} \Delta u_{k+l}^{T} R 1 \Delta u_{k+l} \\
& \text { s.t.: } \quad \dot{x}=f_{d}(x, u), \quad y=g(x, u) \\
& e_{k}=\left(y_{\text {meas }}-y_{\text {pred }}\right)_{k} \\
& \hat{y}_{k+j}=y_{\text {pred }, k+j}+e_{k}
\end{aligned}
$$

where $u, y, x$ are the manipulated, the controlled and the state variables, $y_{\text {meas }}, y_{\text {pred }}, y_{s p}$ are the predicted, the measured variables and the desired set-points and $Q, R$ are the output tracking and the input move weights. The minimization of functional $J(\mathrm{Eq}(1))$ is subject to constraints of $u$ and $y$.

In this work, two MPC-based strategies are combined in a unified control framework in order to take advantage of their synergistic benefits [7]. The first methodology is an online Nonlinear Model Predictive control (NMPC) strategy, which is very appealing due to its ability to handle dynamic nonlinearities of the process under consideration [6]. The second methodology is an explicit or multi-parametric Model Predictive Control (mpMPC) strategy. The mpMPC can provide the optimal solution in real-time, as the solution is computed offline and can be implemented online by simple look-up functions [8]. Both approaches handle state and input constraints and satisfy operating objectives. These characteristics in combination with the tight operation of a PEMFC, signify that an advanced control framework is a suitable approach.

\section{INTEGRATED ONLINE ENERGY MANAGEMENT FRAMEWORK}

Overall the online energy management framework used in this work, has a number of interacting entities that form a computer-aided platform for the monitoring and control of the PEMFC unit [9]. The information flow, from the $\mathrm{I} / \mathrm{O}$ field to the end user, is shown at Fig. 3. Furthermore, Fig. 3 illustrates the interconnection of the control system's entities and the I/O field including the conceptual flow of information from the signal acquisition to the final presentation at the user level through the development of a graphical interface.

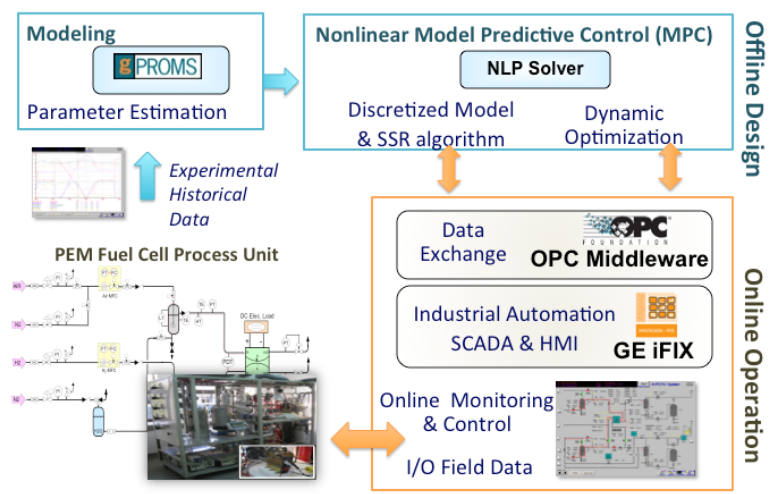

Figure 3. Information flow of the Energy Management Framework of CERTH/CPERI's PEMFC unit

The MPC framework is deployed online to the fuel cell unit using a custom made OPC-based interface that was developed for the communication between the optimizer and the SCADA system [8]. The developed framework has the following desired features:

- Fast calculation of the optimal control actions while taking into account the physical and operating constraints.

- Flexibility to adapt to changing fuel cell response under the influence of disturbances or during start-up and shutdown.

- Incorporation of a multitude of performance criteria under strict computational time demands.

- Easily deployable and maintainable control and optimization solutions.

As far as the MPC related parameters are concerned, the prediction horizon is selected to be sufficiently long with respect to the dynamics of the fuel cell $\left(T_{p}=5 \mathrm{sec}\right)$ divided into $N_{p}$ intervals. The control horizon $\left(T_{c}\right)$ was set to be equal to the sampling time of the SCADA system $(500 \mathrm{~ms})$ and it is divided into $N_{c}$. Finally the selected optimization method is the direct transcription using a reduced gradient NLP solver. The nonlinear fuel cell model is discretized based on OCFE. More specifically there are 10 finite elements $(N E)$ with 4 collocation points $\left(N_{c o p}\right)$ each. 


\section{PEM FUEL CELL EXPERIMENTAL RESULTS}

Overall the objectives for the control system are to effectively address the issue of power generation in an optimum manner. In this context the optimality is defined by the following three terms:

- Operation at a safe region regardless of the load fluctuations.

- Minimization of the fuel consumption and air supply.

- Maintenance of stable temperature conditions ensuring proper gas humidification.

The safe operation is maintained by controlling the reactants at a certain excess ratio level in order to avoid starvation caused by sub-stoichiometric reaction conditions at the cathode and the anode. The safe operating region for the cathode and the anode is defined by two unmeasured variables, the oxygen and hydrogen excess ratios $\left(\lambda_{\mathrm{O} 2}, \lambda_{\mathrm{H} 2}\right)$, expressed as the ratios of the input flow of each gas to the consumed quantities per unit time due to the reaction [10]:

$$
\begin{aligned}
& \lambda_{\mathrm{O} 2}=\frac{\dot{m}_{O 2, c a c h, i n}}{\dot{m}_{O 2, c a G D L}} \\
& \lambda_{H 2}=\frac{\dot{m}_{H 2, a n c h, i n}}{\dot{m}_{H 2, a n G D L}}
\end{aligned}
$$

where $\dot{m}_{O 2, a a c h, i n}, \dot{m}_{H 2, \text { anch,in }}$ are the oxygen and hydrogen input flows at the channels while $\dot{m}_{O 2, a a G D L}, \dot{m}_{H 2, a n G D L}$ are the respective reacted quantities. In order to reach the required excess ratio set-point the air and hydrogen flows $\left(\dot{m}_{\text {air }}, \dot{m}_{H_{2}}\right)$ are used as manipulated variables. The safety of the operation is ensured by maintaining the excess ratios above one $\left(\lambda_{O 2, S P}>1, \lambda_{H 2, S P}>1\right)$.

The power generation and the starvation avoidance objective can be achieved by control actions that aim at an accurate set-point tracking of $P_{S P}, \lambda_{O 2, S P}, \lambda_{H 2, S P}$. On the other hand the temperature control $\left(T_{f c, S P}\right)$ involves two mutually exclusive subsystems, one for the heat-up and another for the cooling

\subsection{Online PEMFC Operation}

An indicative result form the online operation of the PEMFC is presented. The response of the MPC strategy to various power demands was studied at specific operating conditions of temperature and pressure ( $T=338 \mathrm{~K}, P t=1$ bar) is shown in this section. The power modifications were within a range of $1 \mathrm{~W}$ to $5 \mathrm{~W}$, which covers the operational range of the system. The power response to the corresponding control actions of the manipulated variables (Fig. 4,5) as the power demand changes while the oxygen and hydrogen excess ratio remains at a constant set-point $\left(\lambda_{O 2, s p}=3, \lambda_{H 2, s p}=2.5\right)$.

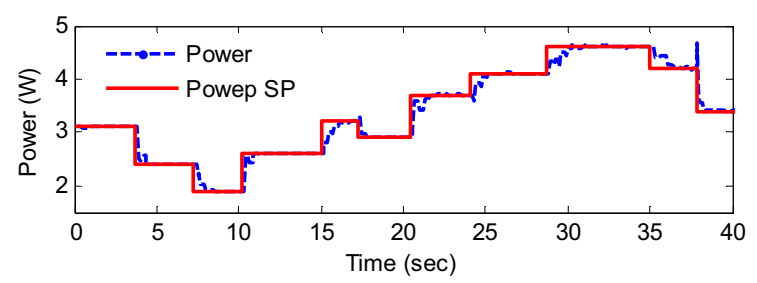

Figure 4. Power demand changes and delivered power by the NMPC controller
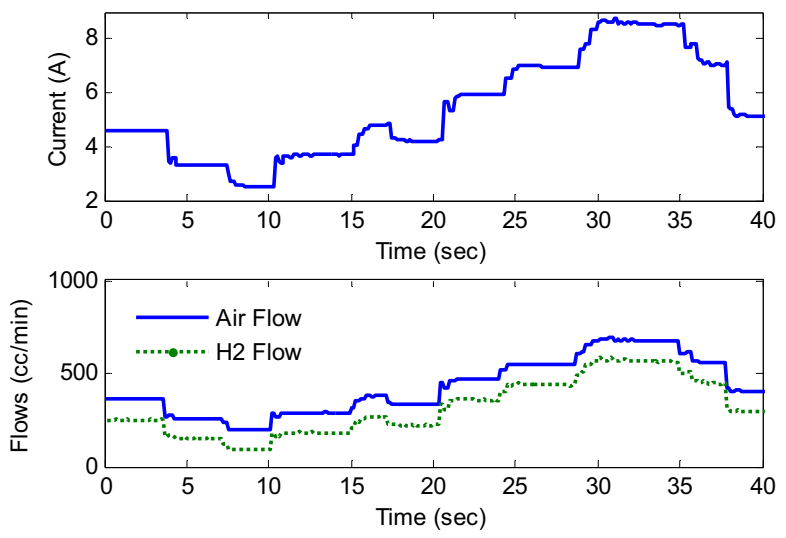

Figure 5. Control actions for the manipulated variables of (a) Current and (b) air and hydrogen mass flows derived by the NMPC controller

The NMPC controller is able to control the fuel cell power to any admissible set-point while maintaining the required excess ratio level.

The mean squared error (MSE) for each objective and some performance metrics are presented at Tab 1 .

Table 1. Performance of the Online NMPC framework

\begin{tabular}{ll}
\hline MSE from Set-point & Performance Metrics \\
\hline Power: $20 \mathrm{~mW}$ & Max. opt. time: $419 \mathrm{~ms}$ \\
Temperature: $0.6^{\circ} \mathrm{C}$ & Average opt. time (SP change): $320 \mathrm{~ms}$ \\
$\lambda_{O 2}: 1.5^{*} 10^{-3}$ & Average opt. time (steady state): $275 \mathrm{~ms}$ \\
$\lambda_{H 2}: 2.1 * 10^{-3}$ & \\
\hline
\end{tabular}

The NMPC controller that was deployed to the PEMFC system can efficiently address the issues of power generation in a safe and controlled manner. Overall, it has been clearly illustrated that the NMPC framework is able to deal with uncertainties and achieve trajectory tracking in a satisfactory manner. Also, the NMPC controller has computational requirements that satisfy the time sampling interval without convergence failures while satisfying realistic constraints imposed by the nature of the PEM fuel cell system.

The presented initial results demonstrate that the proposed control method can be applied online at 
realistic time frame and enable the achievement of multiple, often conflicting, objectives in a dynamically changing environment. Furthermore, due to the nonlinear nature of the model the NMPC approach is capable to adjust to the fuel cell degradation that will shift the operation of the system out of the initial area of operation in time.

\section{CONCLUSIONS}

In this work an integrated energy management approach that relies on a MPC strategy is presented using an advanced MPC strategy to address the operation objectives of a PEMFC unit. A synergy that reveals the potential of exploiting at best the intrinsic features of two model-based control methods (explicit and nonlinear MPC) and the handling of an interesting control problem that involves the optimum operation of an experimental PEM fuel cell unit is demonstrated by the proposed framework. A challenging multivariable nonlinear control problem with measured and unmeasured variables that involves concurrently four operation objectives for a PEM fuel cell system is addressed.

The proposed framework is deployed to the experimental fuel cell and it is validated online. The performance of the MPC strategy shows promising behavior in terms of computational demands and at the same time it guarantees that the PEMFC system operates at an optimum manner.

\section{REFERENCES}

1. European Commission (2011). A roadmap for moving to a competitive low carbon economy in 2050, $\operatorname{COM(2011)~112,~Brussels.~}$

2. CSS (2011). The Impact of Control Technology. T. Samad and A.M. Annaswamy (eds.), IEEE Control Systems Society, www.ieeecss.org (Accessed March 2016).

3. Ziogou C., Voutetakis S., Papadopoulou S., Georgiadis M.C. (2011). Modeling, simulation and experimental validation of a PEM fuel cell system, Computers and Chemical Engineering, 35 (9), 1886-1900.

4. Qin, S. and Badgwell, T. (2003). A survey of industrial model predictive control technology. Control Engineering Practice, 11(7), 733-764.

5. Bauer, M., and Craig, I.K. (2008). Economic assessment of advanced process control - A survey and framework. Journal of Process Control, 18 (1), 2-18.
6. Allgöwer F., Findeisen R., Nagy Z. K. (2004). Nonlinear Model Predictive Control: From Theory to Application, J. Chin. Inst. Chem. Engrs., 35 (3), 299-315.

7. Ziogou C., Pistikopoulos E. N., Georgiadis M. C., Voutetakis S., Papadopoulou S., (2013). Empowering the performance of advanced NMPC by multi-parametric programming - An application to a PEM fuel cell system, Industrial Engineering and Chemistry Research, 52 (13), 4863-4873.

8. Bemporad, A., Morari, M., Dua, V., Pistikopoulos, E. N. (2002). The explicit linear quadratic regulator for constrained systems. Automatica, 38, 3-20.

9. Ziogou C., Papadopoulou S., Georgiadis M. C., Voutetakis S. (2013). On-line nonlinear model predictive control of a PEM fuel cell system, Journal of Process Control, 23(4), 483-492.

10. Pukrushpan, J. T., Peng H., and Stefanopoulou, A.G. (2004). Control-oriented modeling and analysis for automotive fuel cell systems. Journal of Dynamic Systems Measurement and ControlTransactions of the ASME, 126(1), 14-25. 